\title{
Effectiveness of Intratympanic Dexamethasone Injection for Tinnitus Treatment: A Systematic Review and Meta-Analysis
}

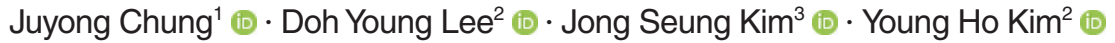 \\ ${ }^{1}$ Department of Otorhinolaryngology, Wonkwang University School of Medicine, Iksan; ${ }^{2}$ Department of Otorhinolaryngology-Head and Neck \\ Surgery, SMG-SNU Boramae Medical Center, Seoul National University College of Medicine, Seoul; ${ }^{3}$ Department of Otolaryngology-Head and \\ Neck Surgery, Chonbuk National University-Chonbuk National University Hospital, Jeonju, Korea
}

Objectives. Intratympanic dexamethasone injection (ITDI) has been introduced as a treatment option for subjective tinnitus. However, the effects of ITDI on patients with tinnitus remain unclear. In the present systematic review and metaanalysis, we evaluated the effectiveness of ITDI for tinnitus treatment.

Methods. We searched Medline, the Cochrane Central Register of Controlled Trials, and Embase. Four double-blind randomized controlled trials that tested the efficacy of ITDI compared with a placebo were deemed eligible for a quantitative meta-analysis, while four prospective studies and seven retrospective studies reporting the effectiveness of ITDI on tinnitus treatment were included in a qualitative synthesis.

Results. In the four studies included in the quantitative meta-analysis, ITDI did not show evidence of tinnitus improvement compared with placebo (odds ratio [OR], 1.38; 95\% confidence interval, 0.53-3.61). In the 11 studies included in the qualitative synthesis, seven retrospective studies without controls reported rates of tinnitus improvement after ITDI ranging from $35.9 \%$ to $91.3 \%$. In the four prospective studies with controls, ITDI seemed to be effective when combined with other drugs for tinnitus treatment.

Conclusion. ITDI alone did not show a significant effect for treating tinnitus compared with placebo. However, the potential of combination treatment of ITDI with other drugs for tinnitus therapy should be further studied in more systematic research.

Keywords. Tinnitus; Injection; Intratympanic; Steroids; Dexamethasone

\section{INTRODUCTION}

Tinnitus involves the perception of sound or sounds in the ear or head without an external source. Most individuals experiencing tinnitus have a neutral reaction to the perception [1]. Although tinnitus may be induced by a peripheral insult such as cochlear

\footnotetext{
- Received July 23, 2021

Revised October 27, 2021

Accepted November 4, 2021

- Corresponding author: Young Ho Kim

Department of Otorhinolaryngology-Head and Neck Surgery, SMG-SNU Boramae Medical Center, Seoul National University College of Medicine, 20 Boramae-ro 5-gil, Dongjak-gu, Seoul 07061, Korea

Tel: +82-2-870-2442, Fax: +82-2-870-3863

E-mail: yhkiment@gmail.com
}

pathology, the structures responsible for generating tinnitus are mainly found centrally, in and around the primary auditory cortex and in many nonauditory higher-order processing centers [2]. Reduced input to the auditory nerve shifts the balance of central excitation and inhibition, which may lead to hyperactivity, increased burst activity, and a more predominant pattern of synchrony [2].

The 2014 American Academy of Otolaryngology-Head and Neck Surgery Foundation Clinical Practice Guidelines for Tinnitus only recommend cognitive behavioral therapy as an evidencebased therapy for persistent, inconvenient tinnitus [3]. These guidelines also recommend against (1) antidepressants, anticonvulsants, anxiolytics, or intratympanic medications; (2) Ginkgo biloba, melatonin, zinc, or other dietary supplements; and (3)

Copyright @ 2022 by Korean Society of Otorhinolaryngology-Head and Neck Surgery.

This is an open-access article distributed under the terms of the Creative Commons Attribution Non-Commercial License (https://creativecommons.org/licenses/by-nc/4.0)

which permits unrestricted non-commercial use, distribution, and reproduction in any medium, provided the original work is properly cited. 
transcranial magnetic stimulation as routine treatment [4]. To date, however, a standard treatment for tinnitus has not been well established, and treatment is often insufficient.

Intratympanic steroid injection (ITSI) is an effective treatment modality for various inner ear diseases such as sudden sensorineural hearing loss and Menière disease. Intratympanic dexamethasone injection (ITDI) has been introduced as a treatment option for subjective tinnitus. In 1982, Sakata et al. [5] first reported the effectiveness of ITDI in patients suffering from cochlear tinnitus. They reported overall good results in $77 \%$ of patients 1 month after ITDI. Additionally, Shulman and Goldstein [6] showed that five of 10 patients experienced tinnitus control for at least 1 year after ITDI. However, in 2005, Araujo et al. [7] published a randomized single-blind study showing that ITDI had no advantage over saline in the treatment of severe, disabling tinnitus. In light of the inconsistent findings described above, the benefits of ITDI in patients with tinnitus remain unclear. In the present meta-analysis, we evaluated the efficacy of ITDI for tinnitus treatment.

\section{MATERIALS AND METHODS}

This systematic review and meta-analysis for prospective and retrospective studies that investigated the efficacy of ITDI in the treatment of tinnitus followed the recommendations of the Preferred Reporting Items for Systematic Reviews and Meta-Analyses (PRISMA) guidelines. A PRISMA flow diagram was used to outline the various phases of the systematic review. This study used quantitative methods to examine the reasons for differences in posttreatment outcomes.

\section{Selection criteria}

We searched Medline, the Cochrane Central Register of Controlled Trials, and Embase up until June 18, 2019. We independently screened the titles and abstracts of all non-duplicated articles and excluded irrelevant titles and abstracts. A final list was agreed upon, and discrepancies regarding the eligibility of studies were resolved by consensus. The following terms were used

\section{H I}

- Intratympanic dexamethasone injection (ITDI) has been used as a treatment option for subjective tinnitus.

- In this systematic review and meta-analysis, we evaluated the effectiveness of ITDI for tinnitus treatment.

- ITDI alone did not show a significant effect for treating tinnitus compared with placebo.

- The potential of combination treatment of ITDI with other drugs for tinnitus therapy should be further studied in more systematic research. for the literature search: (steroid* OR prednisone OR prednisolone OR methylprednisolone OR dexamethasone OR corticosteroid*) AND tinnitus OR intratympanic. We identified additional relevant manuscripts from the references of included studies. Two authors were responsible for retrieving all abstracts, titles, and evaluating related articles.

\section{Inclusion and exclusion criteria}

The inclusion criteria for studies in the review were specified in terms of participants, interventions, comparisons, outcomes, and study design as follows [8]. Studies were included in the systematic review if they (1) involved individuals (adults) with tinnitus; (2) included participants who underwent ITDI; (3) compared the efficacy of ITDI pre- and post-treatment for tinnitus; (4) incorporated relevant outcome measure(s), such as a visual analog scale (VAS), the Tinnitus Handicap Inventory (THI), the Global Improvement scale, or the Tinnitus Awareness Score; and (5) were randomized controlled trials (RCTs), non-RCTs, cohort studies, or case series reporting pre- and post-ITDI results.

We searched for all retrospective studies that tested the efficacy of ITDI in the treatment of tinnitus. The inclusion criteria for our meta-analysis were as follows: (1) studies reporting at least one posttreatment outcome after ITDI, (2) original articles from peer-reviewed scientific journals, and (3) studies with adults. The following types of publications were excluded: (1) animal studies, in vitro studies, review articles, and abstracts, (2) studies without access to original articles (e.g., only abstracts) and/or with incomplete data, and (3) duplicate publications. Additionally, we assessed the risk of bias of the studies included in the qualitative review based on the Newcastle-Ottawa Scale criteria, and all studies showed good or fair quality.

\section{Data extraction}

We reviewed all studies and independently performed data extraction; any discrepancies were resolved by consensus. For each article that reported the outcomes of ITDI, a data collection sheet was created, including data on the following: (1) author and year of publication, (2) study design, (3) otologic diagnosis of the ear with tinnitus, (4) number of patients in study, (5) parameters used to evaluate the outcome of ITDI \& definition of tinnitus improvement, (6) protocol of steroid injection, and (7) tinnitus improvement after ITDI. Values originally provided as percentages were converted back to actual patient numbers for the analysis. Two authors (JC and YHK) extracted the data independently from the included studies and then cross-checked the data. Disagreements were resolved by discussion between the two authors.

\section{Statistical analysis}

Dichotomous data were summarized as odds ratio (OR) with a $95 \%$ confidence interval (CI). If the $95 \%$ CI included a value of 1 , we considered the difference not to be statistically significant. For statistically significant differences in outcomes, the 
number needed to treat was calculated to estimate the overall clinical impact of the intervention. Heterogeneity was quantified using the $\mathrm{I}^{2}$ statistic. We used a fixed-effect model if $\mathrm{I}^{2}<25 \%$ and a random effect model (Dersimonian and Laird method) if $\mathrm{I}^{2}>25 \%$ to combine the results of the studies. Forest plots were used to graphically represent and evaluate the effects of treatment. Publication bias was assessed using a funnel plot and an Egger regression asymmetry test, and a $P<0.1$ in the regression asymmetry test was considered suggestive of publication bias. All statistical analyses in this meta-analysis were performed using RevMan version 5.3 (The Cochrane Collaboration, Oxford, England).

\section{RESULTS}

\section{Characteristics of the studies}

A flow diagram of the initial identification, reasons for exclusion, and final selection of studies is shown in Fig. 1. The search strategy identified 4,473 unique abstracts, including 34 that met the initial screening criteria. After reviewing the full-length articles, 19 studies were excluded due to a lack of relevant data $(n=7)$, unmatched diagnoses $(n=3)$, overlapping data $(n=3)$, review articles $(n=3)$, and unavailability of the full-text article $(n=3)$. Therefore, 15 studies met the inclusion criteria and were included in this systematic review and meta-analysis (Fig. 1). In total, 159 patients (83 who received ITDI vs. 76 who received placebo) were included in the quantitative meta-analysis, and 3,763 patients were included in the qualitative synthesis. The included studies were published between 1983 and 2019.

\section{ITDI versus placebo (intratympanic saline injection) in the quantitative meta-analysis}

Four studies were included in the quantitative meta-analysis of the efficacy of ITDI compared with placebo (Table 1) [7,9-11]. The results of these four studies showed that ITDI did not have a differential effect in reducing the incidence of tinnitus (OR, 1.38; 95\% CI, 0.53-3.61; $P=0.51$ ) compared with placebo (Fig. 2 ). The THI was used in three studies as a parameter for assessing the handicap due to tinnitus [9-11], while one study [7] did not use the THI.

\section{Qualitative synthesis of the efficacy of ITDI for tinnitus treatment} The efficacy of ITDI was investigated in the 11 studies included in the qualitative synthesis (Tables 2 and 3). Of these 11 studies, seven had no control groups (Table 2) [5,12-17], and four had controls (Table 3) [18-21]. Sakata et al. [5] reported the highest efficacy rate $(91.3 \%)$ for ITDI as a treatment for tinnitus. The lowest efficacy rate observed was $35.9 \%$ in the study by Shim et al. [12]. In the studies without controls, the rate of tinnitus improvement ranged from $35.9 \%$ to $91.3 \%$, and the results showed that ITDI seemed to have some effectiveness in tinnitus treatment. Four of the 11 studies were RCTs that tested the efficacy of ITDI compared with other drugs as a control in the treatment of tinnitus (Table 3). The characteristics of the control groups varied from paper to paper. Three studies showed statistically significantly better tinnitus improvement in the ITDI group than in the control group ( $41 \%$ vs. $7 \%, 75.8 \%$ vs. $40.3 \%$, and $67 \%$ vs. $25 \%$, respectively), whereas one study did not (37.5\% vs. $44.0 \%$ ). In two of those three studies, ITDI was used in combination with other drugs for the control of tinnitus and seemed to be effective when combined with other drugs.

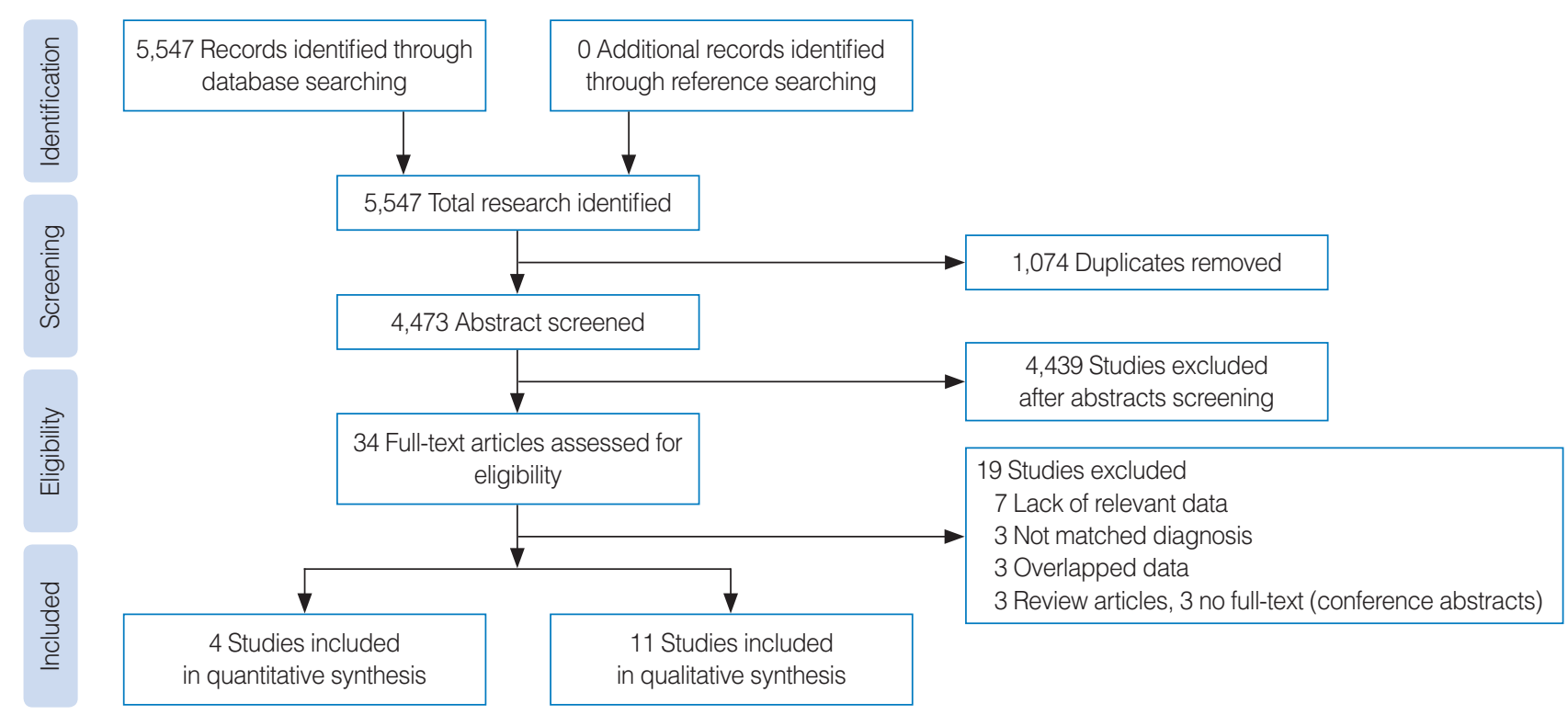

Fig. 1. Preferred Reporting Items for Systematic Reviews and Meta-Analyses (PRISMA) flow diagram outlining the study design. 


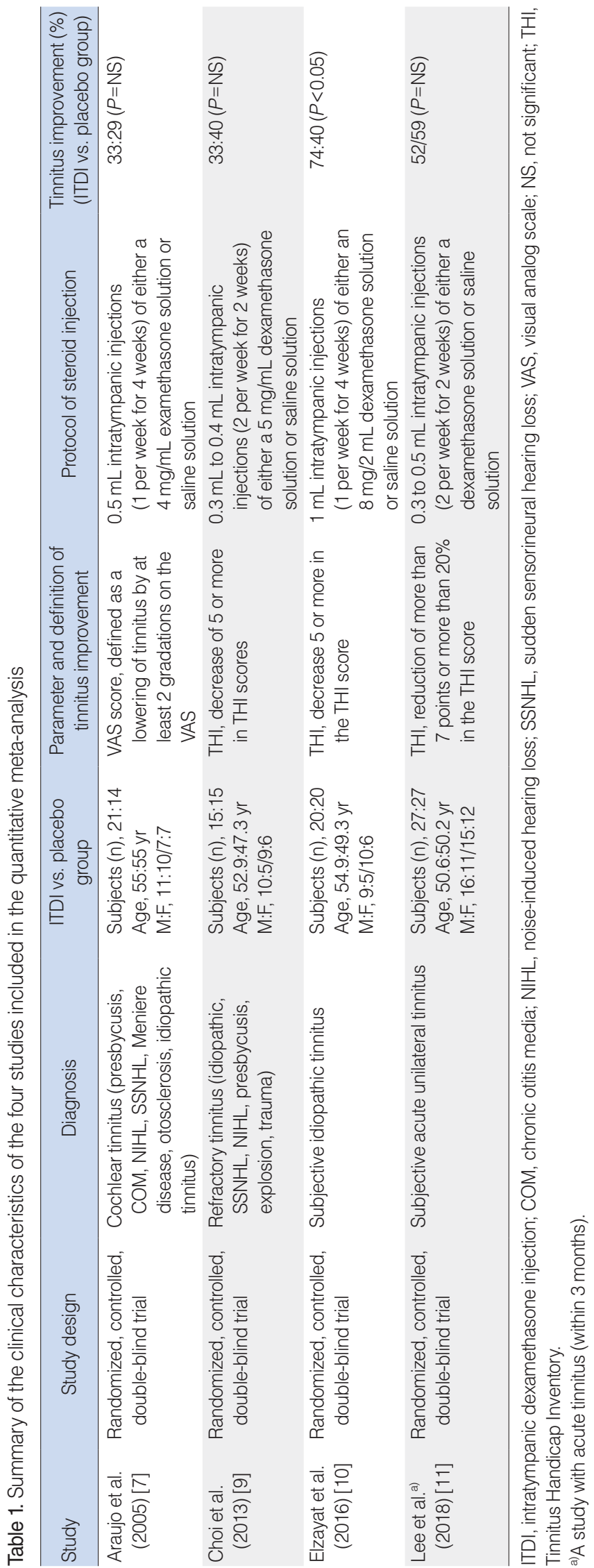

\section{The effectiveness of ITDI in the treatment of acute tinnitus}

We investigated the effectiveness of ITDI for acute tinnitus that had developed within the previous 3 months. In the quantitative meta-analysis and qualitative synthesis, there were five studies of acute tinnitus (Tables 1-3). In three studies that presented comparisons between treatment and control groups, two studies showed statistically significantly better tinnitus improvement in the ITDI group than in the control group $(75.8 \%$ vs. $40.3 \%$ and $67 \%$ vs. $25 \%$, respectively), whereas one study did not ( $52 \%$ vs. $59 \%$ ). In the quantitative meta-analysis for three studies, the results showed that ITDI was not effective in improving acute tinnitus (OR, 2.63; 95\% CI, 0.74-9.33; $P=0.13$ ) (Fig. 3). In the two studies without control groups, the rate of tinnitus improvement in patients who received ITDI was $74.6 \%$ and $35.9 \%$, respectively.

\section{The effectiveness of tinnitus treatment according to the protocol of steroid injection}

We analyzed the protocol of steroid injection in each study. The frequency of steroid injection was usually once or twice a week or every day and the total number of injections was mostly 3 or 4 (Tables 1-3). Of the eight RCTs-four of which were included in the quantitative meta-analysis and four of which were included in the qualitative analysis (Tables 1 and 3) - two studies performed daily injections, and in both of these studies, tinnitus improved significantly after ITDI (2/2). Two of the six RCTs that used steroid injections once or twice a week showed significant improvements after ITDI (2/6). In a meta-analysis comparing the effect size on tinnitus improvement between studies with once or twice weekly injections (OR, 1.48; 95\% CI, 0.65-3.36; $P=0.35)$ and those with daily injections (OR, 4.93; 95\% CI, 2.27-10.70; $P<0.001$ ), patients who received daily injections were significantly more likely to show tinnitus improvement (Supplementary Fig. 1).

\section{DISCUSSION}

Among the various attempts to treat tinnitus, ITDI is clinically burdensome in that it is slightly invasive. Complications such as hearing loss, eardrum perforation, and middle ear inflammation are known to be rare after ITDI [22], but clinicians feel these complications to be more difficult than those after ITDI used to treat other diseases (such as sudden sensorineural hearing loss or Menière disease). In this study, we evaluated the effectiveness of ITDI for tinnitus treatment. Four papers were included in the quantitative meta-analysis, according to which ITDI did not show evidence of tinnitus improvement compared with placebo (Fig. 2). This result demonstrates that ITDI alone may have no therapeutic effect on tinnitus. However, most studies included in the qualitative synthesis suggested that ITDI may contribute to the improvement of tinnitus. Shim et al. [19] reported that combination treatment of ITDI and oral alprazolam was appropriate for 
unilateral acute idiopathic tinnitus within 3 months after onset. Some researchers have suggested that the combination of a benzodiazepine and an endogenous neuroactive steroid can produce both supra-additive anxiolytic effects and infra-additive reinforcing effects [23]. Further RCTs are needed on the synergistic effects of combination steroid and benzodiazepine therapy. In the future, ITDI may have therapeutic applications for tinnitus in limited situations considering various confounding factors, but more objective comparative research is required.
Several noncontrolled studies have demonstrated positive effects of ITDI on the management of tinnitus. Sakata et al. [24] reported that tinnitus improved in $75 \%$ of 3,978 ears immediately after ITDI and in $68 \%$ after 6 months. Cesarani et al. [15], who treated 54 patients with tinnitus using ITDI, demonstrated that complete resolution of tinnitus was observed in five of 50 patients $(10.0 \%)$, and 17 of $50(34.0 \%)$ reported a significant decrease of tinnitus 6 weeks after treatment. However, no controlled study has shown significantly better outcomes than those

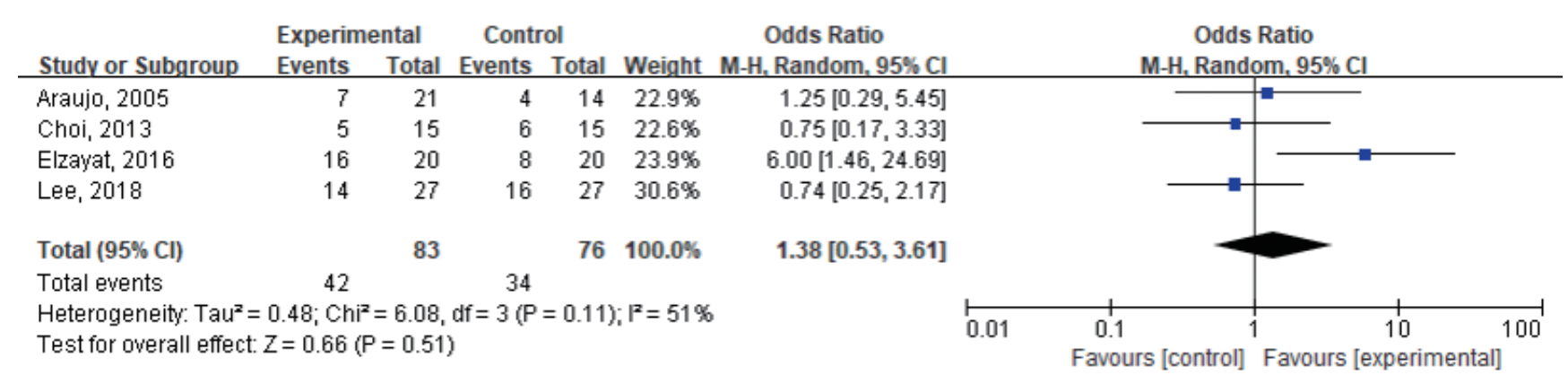

Fig. 2. Forest plot of the meta-analysis for intratympanic dexamethasone injection versus placebo. $\mathrm{Cl}$, confidence interval.

Table 2. Summary of the clinical characteristics of the seven studies without controls included in the qualitative synthesis

\begin{tabular}{|c|c|c|c|c|c|c|}
\hline Study & $\begin{array}{l}\text { Study } \\
\text { design }\end{array}$ & Diagnosis & $\begin{array}{l}\text { ITDI vs. placebo } \\
\text { group }\end{array}$ & $\begin{array}{c}\text { Parameter and definition of tinnitus } \\
\text { improvement }\end{array}$ & $\begin{array}{l}\text { Protocol of steroid } \\
\text { injection }\end{array}$ & $\begin{array}{c}\text { Tinnitus } \\
\text { improvement (\%) }\end{array}$ \\
\hline $\begin{array}{l}\text { Sakata et al. } \\
\text { (1983) [5] }\end{array}$ & $\begin{array}{l}\text { Case } \\
\text { series }\end{array}$ & $\begin{array}{l}\text { Subjective } \\
\text { tinnitus }\end{array}$ & $\begin{array}{l}\text { Subjects }(n), 109 \\
(138 \text { ears) } \\
\text { Age, NA } \\
\text { M:F, NA }\end{array}$ & $\begin{array}{l}\text { Tinnitus was abolished and considerably } \\
\text { ameliorated. }\end{array}$ & - & 91.3 \\
\hline $\begin{array}{l}\text { Kokubu et al. } \\
\text { (1985) [13] }\end{array}$ & $\begin{array}{l}\text { Case } \\
\text { series }\end{array}$ & $\begin{array}{l}\text { Subjective } \\
\text { tinnitus }\end{array}$ & $\begin{array}{l}\text { Subjects (n), } 166 \\
\text { Age, NA } \\
\text { M:F, NA }\end{array}$ & $\begin{array}{l}\text { Tinnitus disappeared and improved } \\
\text { considerably. }\end{array}$ & - & 40.3 \\
\hline $\begin{array}{l}\text { Sakata et al. } \\
\text { (1997) [14] }\end{array}$ & $\begin{array}{l}\text { Case } \\
\text { series }\end{array}$ & $\begin{array}{l}\text { Cochlear } \\
\text { tinnitus }\end{array}$ & $\begin{array}{l}\text { Subjects }(n), 3,041 \\
(3,978 \text { ears }) \\
\text { Age, NA } \\
\text { M:F, NA }\end{array}$ & $\begin{array}{l}\text { VAS score, a level of 0-2 for residual } \\
\text { symptoms represented good effects, } \\
\text { 3-6 represented fair effects. }\end{array}$ & $\begin{array}{l}\text { Dexamethasone injections } \\
\text { ( } 2 \text { or } 4 \mathrm{mg} \text { ), four times at an } \\
\text { interval of } 1-2 \text { weeks }\end{array}$ & 68 \\
\hline $\begin{array}{l}\text { Cesarani et al. } \\
\text { (2002) [15] }\end{array}$ & $\begin{array}{l}\text { Case } \\
\text { series }\end{array}$ & $\begin{array}{l}\text { Subjective } \\
\text { idiopathic } \\
\text { tinnitus }\end{array}$ & $\begin{array}{l}\text { Subjects (n), } 54 \\
\text { Age, } 49.6 \text { yr } \\
M: F, 31: 23\end{array}$ & $\begin{array}{l}\text { VAS score, complete resolution for grade } \\
0 \text { (100\% decrease) and good decrease } \\
\text { for grade } 2 \text { ( } 80 \% \text { decrease) }\end{array}$ & $\begin{array}{l}4 \text { mg dexamethasone } \\
\text { injections, three times monthly } \\
\text { at an interval of } 1 \text { week for } \\
3 \text { consecutive months }\end{array}$ & 74 \\
\hline $\begin{array}{l}\text { Karabulut et al. } \\
\text { (2009) [16] }\end{array}$ & $\begin{array}{l}\text { Case } \\
\text { series }\end{array}$ & $\begin{array}{l}\text { Cochlear } \\
\text { tinnitus }\end{array}$ & $\begin{array}{l}\text { Subjects (n), } 18 \\
\text { Age, NA } \\
\text { M:F, NA }\end{array}$ & $\begin{array}{l}\text { VAS score } \\
\text { THI }\end{array}$ & $\begin{array}{l}0.5 \mathrm{~mL} \text { dexamethasone } \\
(4 \mathrm{mg} / \mathrm{mL}) \text { injections, three } \\
\text { times every other day }\end{array}$ & $\begin{array}{l}\text { Statistically } \\
\text { significant } \\
(P<0.05)\end{array}$ \\
\hline $\begin{array}{l}\text { An et al. }{ }^{\text {a) }} \\
\text { (2014) [17] }\end{array}$ & $\begin{array}{l}\text { Case } \\
\text { series }\end{array}$ & $\begin{array}{l}\text { Acute } \\
\text { subjective } \\
\text { tinnitus }\end{array}$ & $\begin{array}{l}\text { Subjects }(n), 114 \\
\text { Age, } 52.5 \mathrm{yr} \\
\text { M:F, } 40: 74\end{array}$ & $\begin{array}{l}\text { Gl scale: Gl grade >4 (on an 8-grade } \\
\text { scale) } \\
\text { VAS score: >2-point decrease for tinnitus } \\
\text { loudness } \\
\text { Tinnitus awareness score: }>10 \% \text { decrease } \\
\text { THI: >20-point decrease }\end{array}$ & $\begin{array}{l}0.4 \text { to } 0.6 \mathrm{~mL} \text { dexamethasone } \\
(5 \mathrm{mg} / \mathrm{mL}) \text { injections, four } \\
\text { times on } 4 \text { consecutive days }\end{array}$ & 74.6 \\
\hline $\begin{array}{l}\text { Shim et al. }{ }^{\text {a) }} \\
\text { (2017) [12] }\end{array}$ & $\begin{array}{l}\text { Case } \\
\text { series }\end{array}$ & $\begin{array}{l}\text { Acute } \\
\text { idiopathic } \\
\text { tinnitus }\end{array}$ & $\begin{array}{l}\text { Subjects }(n), 39 \\
\text { Age, } 53.56 \mathrm{yr} \\
\text { M:F, 13:26 }\end{array}$ & $\begin{array}{l}\text { Gl: Gl grade }>4 \text { (on an 8-grade scale) } \\
\text { VAS score: }>2 \text {-point decrease for tinnitus } \\
\text { loudness } \\
\text { Tinnitus awareness score: }>10 \% \text { decrease } \\
\text { THI: } 20 \text {-point decrease }\end{array}$ & $\begin{array}{l}0.4 \text { to } 0.6 \mathrm{~mL} \text { dexamethasone } \\
(5 \mathrm{mg} / \mathrm{mL}) \text { injections, four } \\
\text { times on } 4 \text { consecutive days }\end{array}$ & 35.9 \\
\hline
\end{tabular}

ITDI, intratympanic dexamethasone injection; NA, not available; VAS, visual analog scale; THI, Tinnitus Handicap Inventory; GI, Global Improvement. a) A study with acute tinnitus (within 3 months). 


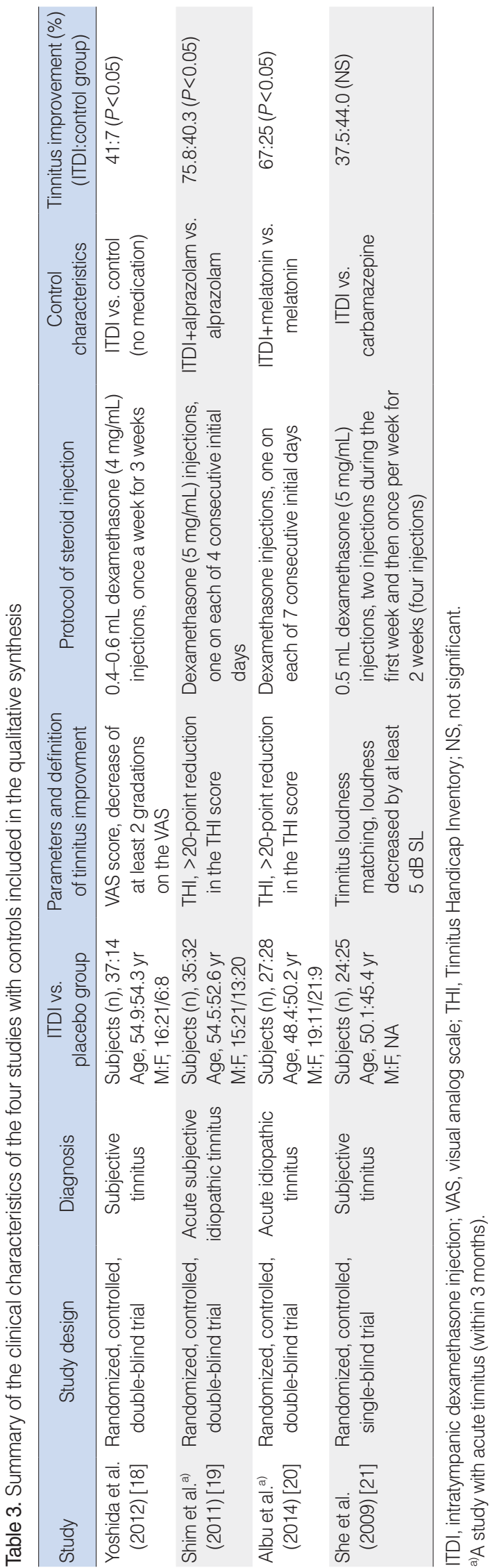

obtained using placebo. Araujo et al. [7] showed no difference between intratympanic injections using dexamethasone and normal saline in the treatment of severe disabling tinnitus; the duration of tinnitus was greater than 1 year in $96 \%$ of the enrolled subjects, and the early improvement rate was $29 \%$ in the control group ( $\mathrm{n}=14$ ears) and $33 \%$ in the study group ( $\mathrm{n}=21$ ears). In another controlled study with 79 patients (84 ears), at 6 months of follow-up after treatment, the effectiveness in the ITDI group was not superior to that in the control group, which received carbamazepine alone [21]. Those authors reported that the improvement rates after intratympanic prednisolone and dexamethasone injections were $45.7 \%$ and $29.2 \%$, respectively, while that for oral carbamazepine was $36.0 \%$ [21].

The mechanism of action of ITSI in the management of tinnitus is still unclear [24]. Steroids are known to exert their effects by penetrating into the inner ear by diffusion through the round window membrane (RWM) and binding with the steroid receptors found in the inner ears of both animals and humans [25]. The putative mechanisms of steroids in treating tinnitus may include suppression of irritability or hypersensitivity of the sensory cells in the inner ear due to damage of cochlear hair cells [26], reduction of the inflammation caused by immune-mediated or autoimmune dysfunction in the inner ear, increase of inner ear vascularity, and/or direct effects on the inner ear neuroepithelium [26].

In the quantitative meta-analysis and qualitative synthesis, most studies (8/15) selected the THI as the main measurement tool for evaluation of tinnitus improvement. Four studies used the VAS alone as a tool for the initial assessment of tinnitus. In five studies, the severity of tinnitus was assessed using the THI alone. Three studies used both the VAS and THI. The THI was developed by Newman et al. [27] in 1996. Since 2000, the THI has been the main evaluation tool for tinnitus, and it was used in nine out of 15 studies. It has been demonstrated that the THI is a brief, easily administered, and reliable measure to evaluate the impact of tinnitus on daily living and the outcomes of medical, surgical, and/or rehabilitative interventions [28]. Moreover, this inventory has shown excellent clinical usefulness for detecting changes in self-perceived tinnitus handicap following interventions such as ITDI [28]. The definition of tinnitus improvement differed from study to study. In terms of the VAS score, tinnitus improvement was defined as a reduction by at least two gradations on the VAS $[7,18]$. Because we tried to investigate the effectiveness of ITDI for tinnitus control in the meta-analysis, the definition of tinnitus improvement using THI scores or tinnitograms could not be standardized. Furthermore, changes in THI scores or the loudness of tinnitus on tinnitograms were not monitored and analyzed for a long period in most studies. Usually, tinnitus improvement was defined as a reduction in the THI score of 5 or more points $[9,10]$. However, a study used a definition of a reduction of more than 7 points or more than $20 \%$ in the THI score [11] and other studies defined tinnitus improve- 


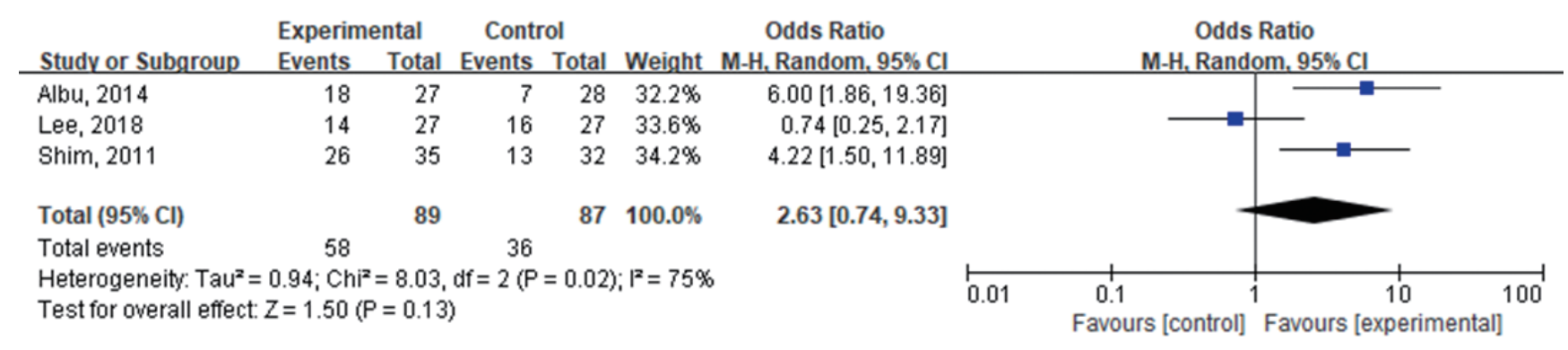

Fig. 3. Forest plot of the meta-analysis for the effectiveness of intratympanic dexamethasone injection in the treatment of acute tinnitus. Cl, confidence interval.

ment as a $>20$-point reduction in THI [12,17,19,20]. Tinnitus loudness matching was defined as a decrease in loudness by at least $5 \mathrm{~dB}$ SL [21]. Since the parameters for evaluating tinnitus and the definitions of tinnitus improvement were different from paper to paper, it was difficult to integrate the parameters and consistently define tinnitus improvement.

This study has a few limitations. First, only four published articles that reported the effectiveness of ITDI compared with placebo for treating tinnitus were included in the quantitative metaanalysis. To ensure the quality of the present research, only double-blind RCTs were selected, and only studies comparing ITDI and placebo were included to unify the characteristics of the experimental and control groups. The sample size was relatively small, and thus, our results regarding the efficacy of ITDI compared with placebo are inconclusive because of the wide CIs. Further studies with larger sample sizes are needed in the future. Second, each study had a different tinnitus duration. Three of the four RCTs in the quantitative meta-analysis enrolled patients who had tinnitus for more than 3 months. In reports by Cesarani et al. [15] and Shea and Ge [29], better ITSI results were obtained in patients with a shorter duration of tinnitus, especially when the treatment was started within 3 months of tinnitus onset. An et al. [17] sought to identify prognostic factors for the outcomes of ITDI in patients with acute subjective tinnitus and demonstrated that the duration of tinnitus was the only variable significantly associated with the cure rate. Therefore, the duration of tinnitus should be considered in tinnitus treatment. An early intervention may be an important factor in the therapeutic efficacy of tinnitus treatment. Among the articles included in the qualitative synthesis, Shim et al. [19] and Albu and Chirtes [20] demonstrated that ITDI combined with other medications (alprazolam and melatonin, respectively) showed effectiveness in improving the development of acute idiopathic unilateral tinnitus within 3 months. However, the article of Lee et al. [11], which was included in the quantitative meta-analysis, reported that ITDI might not be more effective than placebo in the treatment of acute unilateral tinnitus. Although the mechanism of tinnitus in the central auditory system remains unclear, some authors agree that tinnitus is initially triggered by cochlear damage in almost all patients $[4,30,31]$. Moreover, there might be a short therapeutic window to reverse cochlear damage, and if the initiation of ITDI is rapid enough to reverse cochlear damage and subsequent changes in the central auditory system, elimination or management of tinnitus may be possible. Therefore, the timing of treatment initiation has also been reported to be an important factor related to the cure of tinnitus $[17,19]$. In addition, we compared the effect of injection time intervals of ITDI in patients with tinnitus. Patients who received ITDI every day showed significantly better improvement of tinnitus than those who received ITDI once or twice a week. Considering the drug dynamics of steroids, which have rapid clearance rates, repetitive injections with short intervals can maintain a higher steroid concentration in the cochlea [32]. Therefore, an ITDI protocol with daily interventions may be considered as an option for better improvement of tinnitus.

The effect of hearing loss could not be ruled out in the current study. Depending on its cause, tinnitus may be related to hearing loss. Of the 15 papers included in the meta-analysis and systemic review, only nine papers described hearing thresholds. Four articles studied tinnitus patients with normal hearing, while four papers described patients with mild hearing loss and one article analyzed patients with moderate to severe hearing loss. Although the current study mostly included cases of subjective idiopathic tinnitus showing normal hearing, further research is needed to rule out confounding factors such as the degree of hearing loss.

Additionally, fibrosis or ossification around the RWM, which may cause poor permeability of the RWM, may affect the efficacy of ITDI. Silverstein et al. [33] reported the results of round window niche examinations in 41 patients with middle ear endoscopy before intratympanic infusion. Of the 41 patients, seven had partial obstruction of the RWM, and five had complete obstruction. Furthermore, Nordang and colleagues also revealed that hydrocortisone could cause functional obstruction during the course of intratympanic injection treatment by thickening the RWM [34]. Further studies will be needed to address this factor related to fibrosis or ossification around the RWM.

ITDI alone did not show a significant effect for treating tinnitus compared with placebo. However, the potential of combination treatment of ITDI with other drugs for tinnitus control should be further studied through more systematic research to identify 
therapeutic evidence for tinnitus.

\section{CONFLICT OF INTEREST}

No potential conflict of interest relevant to this article was reported.

\section{ACKNOWLEDGMENTS}

This paper was supported by Wonkwang University in 2019.

\section{ORCID}

Juyong Chung

https://orcid.org/0000-0001-5099-5243

Doh Young Lee

https://orcid.org/0000-0003-1590-8559

Jong Seung Kim

https://orcid.org/0000-0002-1384-6799

Young Ho Kim

https://orcid.org/0000-0001-7328-5068

\section{AUTHOR CONTRIBUTIONS}

Conceptualization: JC, YHK. Data curation: JC, DYL. Formal analysis: JC, DYL, JSK. Project administration: JC, YHK. Writing-original draft: JC, YHK. Writing-review \& editing: all authors.

\section{SUPPLEMENTARY MATERIALS}

Supplementary materials can be found via https://doi.org/10. 21053/ceo.2021.01459.

\section{REFERENCES}

1. Cima RF, Mazurek B, Haider H, Kikidis D, Lapira A, Norena A, et al. A multidisciplinary European guideline for tinnitus: diagnostics, assessment, and treatment. HNO. 2019 Mar;67(Suppl 1):10-42.

2. Haider HF, Bojic T, Ribeiro SF, Paco J, Hall DA, Szczepek AJ. Pathophysiology of subjective tinnitus: triggers and maintenance. Front Neurosci. 2018 Nov;12:866.

3. Tunkel DE, Bauer CA, Sun GH, Rosenfeld RM, Chandrasekhar SS, Cunningham ER Jr, et al. Clinical practice guideline: tinnitus. Otolaryngol Head Neck Surg. 2014 Oct;151(2 Suppl):S1-S40.

4. Levine RA. Somatic (craniocervical) tinnitus and the dorsal cochlear nucleus hypothesis. Am J Otolaryngol. 1999 Nov-Dec;20(6):351-62.

5. Sakata E, Itoh A, Ohtsu K, Nakazawa H, Iwashita, Noguchi T. Treatment of cochlear tinnitus: effect of transtympanic infusion with dexamesasone fluid. Audiol Japan. 1983;26(2):148-51.

6. Shulman A, Goldstein B. Intratympanic drug therapy with steroids for tinnitus control: a preliminary report. Int Tinnitus J. 2000;6(1): 10-20.
7. Araujo MF, Oliveira CA, Bahmad FM Jr. Intratympanic dexamethasone injections as a treatment for severe, disabling tinnitus: does it work? Arch Otolaryngol Head Neck Surg. 2005 Feb;131(2):113-7.

8. Moher D, Liberati A, Tetzlaff J, Altman DG; PRISMA Group. Preferred reporting items for systematic reviews and meta-analyses: the PRISMA statement. PLoS Med. 2009 Jul;6(7):e1000097.

9. Choi SJ, Lee JB, Lim HJ, In SM, Kim JY, Bae KH, et al. Intratympanic dexamethasone injection for refractory tinnitus: prospective placebo-controlled study. Laryngoscope. 2013 Nov;123(11):2817-22.

10. Elzayat S, El-Sherif H, Hegazy H, Gabr T, El-Tahan AR. Tinnitus: evaluation of intratympanic injection of combined lidocaine and corticosteroids. ORL J Otorhinolaryngol Relat Spec. 2016 Jun;78(3): 159-66.

11. Lee HJ, Kim MB, Yoo SY, Park SN, Nam EC, Moon IS, et al. Clinical effect of intratympanic dexamethasone injection in acute unilateral tinnitus: a prospective, placebo-controlled, multicenter study. Laryngoscope. 2018 Jan;128(1):184-8.

12. Shim HJ, Lee ES, An YH, Kim DH. Comparison of long-term outcome of intratympanic dexamethasone therapy between acute noise-induced tinnitus and acute idiopathic tinnitus. J Int Adv Otol. 2017 Apr;13(1):53-60.

13. Kokubu M. Transtympanic injection of steroid for tinnitus. Oto-Rhino-Laryngology Tokyo. 1985;28(1):25-30.

14. Sakata E, Ito Y, Itoh A. Clinical experiences of steroid targeting therapy to inner ear for control of tinnitus. int tinnitus J. 1997;3(2):11721.

15. Cesarani A, Capobianco S, Soi D, Giuliano DA, Alpini D. Intratympanic dexamethasone treatment for control of subjective idiopathic tinnitus: our clinical experience. Int Tinnitus J. 2002;8(2):111-4.

16. Karabulut H, Acar B, Babademez MA, Tuncay S, Karasen RM. Intratympanically dexamethasone injection application effects as a treatment of tinnitus. Anatol J Clin Investig. 2009;3(3):154-8.

17. An YH, Yu KK, Kwak MY, Yoon SW, Shim HJ. Prognostic factors for the outcomes of intratympanic dexamethasone in the treatment of acute subjective tinnitus. Otol Neurotol. 2014 Sep;35(8):1330-7.

18. YoshidaT,Teranishi M, Iwata T, Otake H, Nakashima T. Intratympanic injection of dexamethasone for treatment of tinnitus in patients with sudden sensorineural hearing loss. Audiol Res. 2012 Jan;2(1):e2.

19. Shim HJ, Song SJ, Choi AY, Hyung Lee R, Yoon SW. Comparison of various treatment modalities for acute tinnitus. Laryngoscope. 2011 Dec;121(12):2619-25.

20. Albu S, Chirtes F. Intratympanic dexamethasone plus melatonin versus melatonin only in the treatment of unilateral acute idiopathic tinnitus. Am J Otolaryngol. 2014 Sep-Oct;35(5):617-22.

21. She W, Dai Y, Du X, Chen F, Ding X, Cui X. Treatment of subjective tinnitus: a comparative clinical study of intratympanic steroid injection vs. oral carbamazepine. Med Sci Monit. 2009 Jun;15(6):PI35-9.

22. Kim YH, Lee DY, Lee DH, Oh S. Tympanic membrane perforation after intratympanic steroid injection: a systematic review and metaanalysis. Otolaryngol Head Neck Surg. 2022 Feb;166(2):249-59.

23. Gunter BW, Platt DM, Rowlett JK. Differential interactions engendered by benzodiazepine and neuroactive steroid combinations on schedule-controlled responding in rats. Pharmacol Biochem Behav. 2015 Oct;137:53-9.

24. Sakata $\mathrm{E}$, Itoh $\mathrm{A}$, Itoh $\mathrm{Y}$. Treatment of cochlear-tinnitus with dexamethasone infusion into the tympanic cavity. Int Tinnitus J. 1996;2: 129-35.

25. Pitovski DZ, Drescher MJ, Drescher DG. Glucocorticoid receptors in the mammalian inner ear: RU 28362 binding sites. Hear Res. 1994 Jun;77(1-2):216-20.

26. Yilmaz I, Yilmazer C, Erkan AN, Aslan SG, Ozluoglu LN. Intratympanic dexamethasone injection effects on transient-evoked otoacoustic emission.Am J Otolaryngol. 2005 Mar-Apr;26(2):113-7.

27. Newman CW, Jacobson GP, Spitzer JB. Development of the tinnitus 
handicap inventory. Arch Otolaryngol Head Neck Surg. 1996 Feb; 122(2):143-8.

28. Newman CW, Sandridge SA, Jacobson GP. Psychometric adequacy of the Tinnitus Handicap Inventory (THI) for evaluating treatment outcome. J Am Acad Audiol. 1998 Apr;9(2):153-60.

29. Shea JJ Jr, Ge X. Dexamethasone perfusion of the labyrinth plus intravenous dexamethasone for Meniere's disease. Otolaryngol Clin North Am. 1996 Apr;29(2):353-8.

30. Moller AR. The role of neural plasticity in tinnitus. Prog Brain Res. 2007;166:37-45.

31. Sanchez TG, da Silva Lima A, Brandao AL, Lorenzi MC, Bento RF. Somatic modulation of tinnitus: test reliability and results after re- petitive muscle contraction training. Ann Otol Rhinol Laryngol. 2007 Jan;116(1):30-5.

32. Kwak MY, Yang CJ, Shim HJ, Song CI, Kim JY, Lee IW, et al. Intratympanic steroid injection for sudden sensorineural hearing loss: impact of injection interval on therapeutic efficacy. Auris Nasus Larynx. 2020 Dec;47(6):982-9.

33. Silverstein H, Rowan PT, Olds MJ, Rosenberg SI. Inner ear perfusion and the role of round window patency.Am J Otol. 1997 Sep;18(5): 586-9.

34. Nordang L, Linder B, Anniko M. Morphologic changes in round window membrane after topical hydrocortisone and dexamethasone treatment. Otol Neurotol. 2003 Mar;24(2):339-43. 